\title{
Prenatal exposure to maternal very severe obesity is associated with impaired neurodevelopment and executive functioning in children
}

\author{
Theresia H Mina ${ }^{1,2,5}$, Marius Lahti ${ }^{1,3,5}$, Amanda J Drake ${ }^{1}$, Fiona C Denison ${ }^{2,4}$, Katri Räikkönen ${ }^{3}$, Jane E Norman ${ }^{2,4}$ and \\ Rebecca M Reynolds ${ }^{1,2}$
}

BACKGROUND: Prenatal maternal obesity has been associated with an increased risk of neurocognitive problems in childhood, but there are fewer studies on executive functioning.

METHODS: Tests and questionnaires to assess neurodevelopment, executive functioning, and the ability to delay gratification were conducted in 113 children (mean (SD) = $4.24(0.63)$ years of age) born to mothers with very severe obesity $\left(\mathrm{SO}\right.$, body mass index $\left.(\mathrm{BMI}) \geqslant 40 \mathrm{~kg} / \mathrm{m}^{2}, n=51\right)$ or to lean mothers (BMl $\leqslant 25 \mathrm{~kg} / \mathrm{m}^{2}, n=62$ ).

RESULTS: Prenatal maternal SO predicted poorer neurodevelopment (unstandardized regression coefficient $(B)=-0.42$, 95\% confidence interval (Cl) $(-0.82 ;-0.02))$, worse problemsolving (odd ratio $(\mathrm{OR})=0.60,95 \% \mathrm{Cl}(1.13 ; 0.07)$ ), and fine motor skills (OR=4.91, 95\% Cl $(1.27 ; 19.04))$, poorer executive functioning in areas of attention, inhibitory control, and working memory (standardized $\mathrm{B}=3.75,95 \% \mathrm{Cl}(1.01 ; 13.93)$ ) but not in self-gratification delay. The effects were independent of maternal concurrent psychological well-being and child's BMI, but not independent of maternal education.

CONCLUSION: Future studies should investigate whether perinatal management of maternal obesity could prevent adverse outcomes in child neurodevelopment.

O ne in five women is obese at the start of pregnancy (1), exposing the offspring to a greater risk of childhood obesity (2) and future cardiometabolic problems. In an increasing number of studies, prenatal exposure to maternal obesity has been associated with adverse outcomes for children's neurocognitive development. This is of concern because children with poorer neurocognitive development face greater difficulties in their education and other aspects of life as they grow up (3). Furthermore, lower intelligence in childhood has been associated with a greater risk of mortality and morbidity in adulthood, possibly because of a higher likelihood of adopting an unhealthy lifestyle and other childhood adversities (4).

Prenatal exposure to maternal obesity is associated with a higher risk of intellectual disability $(5,6)$, lower intelligence quotients (7-10), and an increased risk of neurodevelopmental delay (11-15). Increased prevalence of neurocognitive problems including lower verbal, reading, and maths ability has also been reported in children exposed to prenatal maternal obesity (10,16-18), although the findings are inconsistent (19).

The evidence for an effect of maternal obesity on child executive function is more limited. Only two studies have studied children born to obese mothers, and both reported worse self-regulation, inhibitory skills, and decision-making in these children $(10,20)$. However, to our knowledge, no study has investigated delays in self-gratification. In one study that characterized obese children, the association between the ability to delay self-gratification and childhood obesity was weakened after adjusting for maternal body mass index (BMI), implying that maternal BMI is a possible contributing factor to a child's executive function (21).

Whether or not maternal obesity predicts child neurocognitive development independently of familial confounding, including maternal concurrent psychological well-being and child's own obesity, remains uncertain. This is important as maternal psychological distress, which is increased in obese mothers $(22,23)$, has adverse effects on children's cognition (24-26), and also as childhood obesity is correlated with problems in various aspects of neurocognitive development, including impaired executive function, visuospatial performance, and motor skills (27). Other uncertainties include whether or not the maternal obesity effect on child's neurocognitive development, especially intelligence quotient, reflects a mere familial predisposition or whether it is independent of postnatal environment such as maternal

\footnotetext{
'University/BHF Centre for Cardiovascular Sciences, Queen's Medical Research Institute, University of Edinburgh, Edinburgh, Scotland, UK; ${ }^{2}$ Tommy's Centre for Maternal and Fetal Health, Queen's Medical Research Institute, University of Edinburgh, Edinburgh, Scotland, UK; ${ }^{3}$ Institute of Behavioral Sciences, University of Helsinki, Helsinki, Finland; ${ }^{4}$ MRC Centre for Reproductive Health, Queen's Medical Research Institute, University of Edinburgh, Edinburgh, Scotland, UK. Correspondence: Rebecca M. Reynolds (r.reynolds@ed.ac.uk)

${ }^{5}$ Joint first authors.

Received 26 September 2016; accepted 6 February 2017; advance online publication 17 May 2017. doi:10.1038/pr.2017.43
} 
education (28), but a recent sibling analysis concluded that maternal obesity remains a significant predictor of child's intelligence quotient independent of familial confounders (9). Such contrasting literature emphasizes the importance of considering both maternal obesity and the associated sociodemographic covariates.

The current work evaluated neurodevelopmental problems and executive functioning in children exposed to prenatal maternal very severe obesity $\left(\mathrm{SO}, \mathrm{BMI} \geqslant 40 \mathrm{~kg} / \mathrm{m}^{2}\right.$, obese class III) using global developmental screening and two objective tests evaluating self-regulation and delay of self-gratification. We hypothesized that prenatal exposure to maternal SO would be associated with an increased risk of neurodevelopmental delay, and poorer executive functioning including poorer attention, self-inhibition, and delay of self-gratification in early childhood. Further, we hypothesized that these associations would be independent of obesity-linked obstetric confounders, as well as previously overlooked covariates such as maternal concurrent psychological well-being and the child's own current BMI.

\section{METHODS}

\section{Participants}

The participants were children whose mothers participated in a longitudinal study of pregnancy with $\mathrm{SO}\left(\mathrm{BMI} \geqslant 40 \mathrm{~kg} / \mathrm{m}^{2}\right.$ at their first antenatal booking, World Health Organization) and lean controls $\left(\mathrm{BMI} \leqslant 25 \mathrm{~kg} / \mathrm{m}^{2}\right)$ in Scotland, UK, 2008-2013. The study was ethically approved by the West of Scotland Research Ethical Committee (REC: 14/WS/1046, R\&D: 2014/0278), and all mothers gave written informed consent. We screened 357 potential participants (135 lean mothers and 222 mothers with SO) from the pregnancy study for eligibility, and excluded children who had moved out of the area or who were under the Child Protection Register (Scottish register for children who are identified as needing support or protection from all abuses). Of the 324 invited to take part in the study, 113 mother-child dyads (62 lean mothers and 51 mothers with SO) agreed to participate either through a clinic visit and/or completing questionnaires at home. Mothers who declined to participate were more likely to be the ones with SO (70.1\% vs. $45.1 \%$, $P<0.001)$, but they did not differ from the study participants in parity, prevalence of GDM, child's sex, gestational age at delivery, or sex- and gestational age-adjusted child's birth weight (all $P \geqslant 0.30$ ). Table 1 shows the characteristics of the sample according to maternal SO status. Compared with lean mothers, mothers with SO had lower education levels, were more likely to be older, had GDM, and had higher depressive symptoms at follow-up (Table 1).

Evaluating the Delay of Self-Gratification: The Marshmallow Test Children's self-regulation and inhibitory abilities were evaluated using the Marshmallow Test, which assesses the delay of immediate self-gratification for more snack rewards (29). The test has been shown to predict many cognitive and behavioral outcomes in childhood and adulthood, including better cognitive academic competences, concentration, and attentiveness, self-organization, self-control, and resilience (against stress) $(3,29)$. A standardized setup was arranged before the participants' arrival, comprising a low table, a child-friendly low chair, a marshmallow at the center of a white paper plate, no toys, and a camcorder positioned out of sight of the child. Mothers were asked to wait in an ante-chamber with the door left ajar to reduce the possibility of separation distress while their child completed the test, and were discouraged from watching the child's performance. Rather than using the original version of the Marshmallow Test where the children are trained to call the experimenter to return when they want to have the reward (29), a simplified version shown to reduce the potential variability of experimenter's reliability was used (30). Before the test, one marshmallow was presented and the child was told, "You may eat the marshmallow now, but if you wait until I come back, I will give you another one." Assent was obtained, and comprehension was confirmed before leaving the child alone. The child was made to wait for $15 \mathrm{~min}$ (this information was not disclosed to the child). The test started when the nurse had left the room, and ended when the marshmallow was entirely eaten or when 15 min was reached. The time at which the test started and ended was recorded to determine the total duration of the test, with a longer test duration indicating better self-control. The child received a second marshmallow as a reward regardless of the test result. The test was terminated if the children were distressed, unsettled, or continuously attempting to find their mothers. A maximum of three re-trials were allowed before the test was completely annulled (6 children, 3 from each group). There were 79 data sets (43 lean mothers and 36 mothers with SO) available for analysis.

\section{Evaluating Behavioral Self-Regulation: The HTKS Test}

Following a brief break after the Marshmallow Test, the HTKS Test was conducted to evaluate the child's executive functioning components of ability to pay attention, attentional flexibility, working memory, and inhibitory control (31). The test has good inter-rater reliability, internal consistency, concurrent validity, and test-retest reliability (31). The HTKS Test is a short game for 3-8year-olds with three difficulty levels and a maximum of three allowed numbers of repeated instruction/level. Each level has 10 tasks with a score 0 for incorrect response, 1 for "self-corrected", or 2 for "correct"; therefore, the score range is $0-60$. If the child scores $\geqslant 4$ at the previous level, he/she can proceed to the next difficulty level. In this test, children must respond the "opposite" way to that instructed, so they should touch their head if instructed "touch your toes". At first level, two behavioral rules were introduced (head-toe and toehead), and another two new rules (head-toes, toes-head, kneeshoulder, and shoulder-knee) were added at second level. At third level, all of the four behavioral rules (head-knee, knee-head, toesshoulder, and shoulder-toes) were switched. When the child refused to play or did not pass the cutoff mark, the test was halted and no retrials were allowed. The test was completed by 77 children (42 lean mothers and 35 mothers with SO). Total scores as primary outcome were tabulated, with higher scores indicating better executive functioning.

\section{Assessing Neurodevelopment: ASQ}

Mothers completed the ASQ-3 (Brookes Publishing, Baltimore, Maryland), which assesses children's neurocognitive and psychomotor developmental milestones and detects neurodevelopmental delay for the first $5 \frac{1}{2}$ years of life. The ASQ is series of age-specific questions; thus, the developmental tasks conducted on each child are specific to the age group. ASQ-3 is one of the most globally used tests of developmental delay with excellent test-retest reliability, intraobserver reliability, internal consistency, and concurrent validity.

The ASQ contains five to six questions per section for five sections assessing communication, gross motor, fine motor, problem-solving, and personal-social development. The range of scores is $0-60$ for each subscale, and 300 for a sum-score of these subscales as a global measure of neurodevelopment, where higher scores indicate better neurodevelopment and is a recommended scoring method (32). Scores were available from 104 (57 lean mothers and 47 mothers with $\mathrm{SO}$ ) returned questionnaires. As the distributions of the ASQ component scores were highly skewed with at least 31 out of 104 children achieving a full score $=60$ per component, binary variables per component were derived to indicate whether or not each child could complete all the age-specific assigned tasks. The binary ASQ scores were reverse-scored, such that higher number indicates higher neurocognitive or psychomotor developmental difficulties $(0=$ score $=60$ vs. $1=$ score $<60)$. 
Table 1. Mother and child characteristics according to maternal status of severe obesity

\begin{tabular}{|c|c|c|c|}
\hline & Lean $(n=62)$ & SO $(n=51)$ & $P$ value \\
\hline \multicolumn{4}{|l|}{ Maternal characteristics } \\
\hline \multicolumn{4}{|l|}{ Maternal education level, $n(\%)^{a}$} \\
\hline University & $57(91.9)$ & $21(44.7)$ & \multirow{2}{*}{$<0.001^{\mathrm{b}}$} \\
\hline Data missing & 0 & 4 & \\
\hline Ex-smoker or current smoker & $26(41.9)$ & $25(49.0)$ & $0.45^{\mathrm{b}}$ \\
\hline Maternal GDM, $n(\%)$ & $3(4.8)$ & $10(19.6)$ & $0.01^{\mathrm{b}}$ \\
\hline \multicolumn{4}{|l|}{ Parity, $n(\%)$} \\
\hline Nulliparous (0) & $39(62.9)$ & $26(51.0)$ & $0.20^{\mathrm{b}}$ \\
\hline Data missing, $n$ & 1 & 2 & - \\
\hline \multicolumn{4}{|l|}{ Maternal concurrent depressive symptoms } \\
\hline GHQ scores, mean (SD) & $1.2(1.3)$ & $3.3(3.3)$ & $<0.001^{\mathrm{C}}$ \\
\hline Data missing, $n$ & 1 & 2 & - \\
\hline Maternal age, mean (SD) & $31.26(4.49)$ & $33.65(5.57)$ & $0.013^{c}$ \\
\hline \multicolumn{4}{|l|}{ Infant characteristics } \\
\hline Infant's SDS birth weight, mean (SD) & $0.1(1.0)$ & $0.5(1.0)$ & $0.050^{c}$ \\
\hline Infant's gestational age in days, mean (SD) & $281.0(9.8)$ & $279.7(9.7)$ & $0.47^{c}$ \\
\hline \multicolumn{4}{|l|}{ Child sex, $n$ (\%) } \\
\hline Male & $29(46.8)$ & $23(45.1)$ & $0.86^{\mathrm{b}}$ \\
\hline Female & $33(53.2)$ & $28(54.9)$ & \\
\hline \multicolumn{4}{|l|}{ Foreign language spoken at home } \\
\hline Yes, $n(\%)$ & $9(14.5)$ & $10(20.0)$ & $0.44^{\mathrm{b}}$ \\
\hline Data missing, $n$ & 0 & 1 & - \\
\hline Child's age at follow-up in years, mean (SD) & $4.1(0.6)$ & $4.5(0.6)$ & $0.001^{c}$ \\
\hline
\end{tabular}

BMI, body mass index; GDM, gestational diabetes mellitus; GHQ, General Health Questionnaire (minimum-maximum, 0-15); SDS, British standard deviation score; SO, very severe obesity; STAI, State Trait Anxiety Index (minimum-maximum, 0-80).

Bold text: $P \leqslant 0.05$

a'Non-university refers to Scottish Highers, International Baccalaureate, SVQ (Scottish Vocational Qualification), and diploma, whereas 'University' refers to undergraduate, master, and PhD degrees.

${ }^{b}{ }^{2}$-test.

'Student's t-test.

\section{Anthropometric Measurement of Children}

Birth weight was obtained from hospital records, and the child's current weight and height were measured using SECA electronic weighing scale and stadiometer, respectively (SECA, Birmingham, UK). Because of high multicollinearity between child weight and BMI $(r=0.77, P<0.001)$, and child weight and height $(\mathrm{r}=0.75$,
$P<0.001$ ), child height and BMI were used as measures of child anthropometry. Child's anthropometry measures across times were subsequently age- and sex-adjusted using the United Kingdom World Health Organization growth chart (LMS Growth Microsoft excel free add-ins by Pan \& Cole (http://healthforallchildren.com, version 2.77, 2012, Oxford, UK), generating SDS values. Children 


\section{Articles $\mid$ Mina et al.}

Table 2. Associations between prenatal exposure to maternal severe obesity and child's scores in neurodevelopmental tasks

\begin{tabular}{|c|c|c|c|c|c|c|c|c|c|c|}
\hline \multirow[t]{2}{*}{ Neurodevelopment tasks } & \multicolumn{2}{|l|}{ Model 1} & \multicolumn{2}{|l|}{ Model 2} & \multicolumn{2}{|l|}{ Model 3} & \multicolumn{2}{|c|}{ Model 4} & \multicolumn{2}{|c|}{ Model 5} \\
\hline & $B(95 \% \mathrm{Cl})^{\mathrm{a}}$ & $P$ value & $B(95 \% C l)^{a}$ & $P$ value & $B(95 \% \mathrm{Cl})^{\mathrm{a}}$ & $P$ value & $B(95 \% C l)^{\mathrm{a}}$ & $P$ value & $B(95 \% \mathrm{Cl})^{\mathrm{a}}$ & $P$ value \\
\hline $\begin{array}{l}\text { Marshmallow Test, } n=79 \\
\text { ( } 43 \text { lean mothers and } 36 \\
\text { mothers with SO) }\end{array}$ & $-0.45(-0.93 ; 0.03)$ & $\underline{0.06}$ & $-0.27(-0.79 ; 0.24)$ & 0.29 & $-0.28(0.83 ; 0.27)$ & 0.28 & $\begin{array}{c}-0.30(-0.89 \\
0.29)\end{array}$ & 0.32 & $\begin{array}{c}-0.58(-1.25 \\
0.10)\end{array}$ & 0.09 \\
\hline $\begin{array}{l}\text { HTKS Test, } n=77 \\
\text { ( } 42 \text { lean mothersand } 35 \\
\text { mothers with SO) }\end{array}$ & $-0.55(-1.02 ;-0.09)$ & 0.02 & $-0.60(-1.06 ;-0.15)$ & 0.01 & $-0.68(-1.17 ;-0.19)$ & 0.01 & $\begin{array}{c}-0.60(-1.13 \\
0.07)\end{array}$ & 0.03 & $\begin{array}{c}-0.50(-1.10 \\
0.11)\end{array}$ & 0.11 \\
\hline $\begin{array}{l}\text { ASQ sum-score, } n=101 \\
\text { ( } 56 \text { lean mothers and } 45 \\
\text { mothers with SO) }\end{array}$ & $-0.42(-0.82 ;-0.02)$ & 0.04 & $-0.43(-0.86 ; 0.01)$ & $\underline{0.053}$ & $-0.52(-1.00 ;-0.04)$ & 0.03 & $\begin{array}{c}-0.49(-0.99 \\
0.00)\end{array}$ & 0.05 & $\begin{array}{c}-0.37(-0.94 \\
0.21)\end{array}$ & 0.21 \\
\hline
\end{tabular}

ASQ, Ages and Stages; Cl, confidence interval; HTKS, Head-Toe-Knee-Shoulder-Test; SO, severe obesity.

Bold text: $P \leq 0.05$, underlined text: $P \leq 0.1$. Higher scores indicate better performance in neurodevelopmental tasks. All dependent variables are expressed in SD units. Model 1 is adjusted for the age and sex of the child. Model 2 is adjusted for model 1 covariates+maternal gestational diabetes, smoking status during and before pregnancy, age, and foreign language spoken at home. Model 3 is adjusted for model 2 covariates+maternal concurrent anxiety and depressive symptoms at follow-up. Model 4 is adjusted for model 3 covariates+child's SDS birth weight and SDS BMI at follow-up. Model 5 is adjusted for model 4 covariates+maternal education level.

${ }^{a} B(95 \%$ Cl): unstandardized regression coefficient comparing children born to severely obese vs. lean (referent) mothers in linear regression models $1-5$ and $95 \%$ confidence interval of the regression coefficient.

born to mothers with SO had higher SDS birth weight, and, at follow-up, they were significantly older and had increased BMI (Table 1). Seven out of $113(6.36 \%)$ children were obese ( $>98$ th centile or $>2$ SD, Royal College of Paediatrics and Child Health guideline, UK), and six of these were in the SO group.

\section{Sociodemographic, Prenatal, and Concurrent Covariates}

Any covariates with associations with either the predictor (maternal SO status) or the outcome variables (total duration of Marshmallow Test, total scores of HTKS, component, and sum-scores of ASQ) were included. Prenatal covariates were mothers' prenatal smoking status (never smoked and 'others' including ex-smoker or currently smoking), presence of gestational diabetes, infant sex, and infant's SDS birth weight. Concurrent covariates were the child's age at visit, child's SDS BMI, foreign language spoken at home, maternal age, and maternal current anxiety and depressive symptoms assessed with the state anxiety score of Spielberger State Trait Anxiety Index and General Health Questionnaire, respectively. The sociodemographic covariate was maternal education level, which was grouped into 'non-university' (Scottish Highers, International Baccalaureate, Scottish Vocational Qualification, and diploma) and 'university' (undergraduate, master, and $\mathrm{PhD}$ degrees).

\section{Statistical Analysis}

Statistical analyses were performed using SPSS 23.0 (IBM, Armonk, New York). To obtain normal distributions, the scores of state anxiety State Trait Anxiety Index and General Health Questionnaire were square-root-transformed. The total duration of Marshmallow Test and ASQ sum-scores was rank-transformed using Blom's formula. All continuous measures of study outcomes were converted into $z$-scores for comparison of effect sizes.

Pearson $r$ correlation, Student's $t$-test, logistic regression analyses, and $\chi^{2}$-tests were used to determine the associations between the covariates with maternal SO and with the neurocognitive and neurodevelopmental outcome variables.

Multiple linear/logistic regressions were performed using maternal SO status as the independent variable and the child's neurodevelopmental outcomes as the dependent variables. The associations with ASQ sum-score, marshmallow total test duration score, and the HTKS score were examined with linear regressions, whereas the analyses on the five individual ASQ subscales with the binary outcome variables were assessed with logistic regressions. We arranged five different regression models according to the approach to our earlier work as well as others (33). The first regression model (model 1) included infant sex and age at visit as covariates. Model 2 included model $1+$ maternal age, smoking, and GDM status during pregnancy, as these are related to well-being during pregnancy (33), and the use of foreign language at home. This was followed by model 3 , which comprised model $2+$ maternal concurrent anxiety and depressive symptoms, that may bias her estimates of the child or influence the effects through familial confounding (24-26). Model 4 included model 3 + child's SDS birth weight and SDS BMI at visit, which can potentially be in the causal pathway. Finally, model 5 encompassed model $4+$ maternal education status, which is known to heavily influence neurodevelopment (28) and not introduced earlier because of its high multicollinearity. Introducing maternal education toward the final regression models would allow us to better distinguish maternal obesity effect instead of masking its true effect.

To ensure that the findings on the continuous scales were not due to floor or ceiling effects, Tobit regressions were run in parallel using the five models above, with scale-specific and score distributionbased floor value $=0$ for HTKS, ceiling value $=900 \mathrm{~s}$ for total duration of Marshmallow Test, and ceiling value $=300$ for ASQ sumscores. Multiple linear/logistic regressions with confounders in models 1-4 were also performed in a subgroup of children whose mothers attended university to find out whether maternal SO status is a predictor of child's neurodevelopmental independent of maternal education.

\section{RESULTS}

\section{Background Associations with Covariates}

Supplementary Table S1 online illustrates the associations between the covariates and continuous outcome variables, and Supplementary Table S2 illustrates the associations between the covariates and the individual Ages and Stages Questionnaire (ASQ) scales with binary outcomes for all participants. Girls had higher continuous ASQ sum-scores, whereas boys had a higher risk of difficulties in ASQ personal/ social development, fine motor, and problem-solving. Older child age was correlated with higher Head-Toe-KneeShoulder (HTKS) scores. Children born to mothers with lower educational levels had lower ASQ sum-scores and a higher risk of difficulties in ASQ problem-solving and gross motor skills. Children who spoke a foreign language at home had an increased risk of ASQ problem-solving difficulties. Children whose mothers had never smoked had higher HTKS scores and higher ASQ sum-scores than those whose mothers smoked before or during pregnancy. Children whose mothers were older had higher HTKS scores. Higher maternal concurrent anxiety symptoms were associated with lower ASQ sum-score and an increased risk of ASQ communication difficulties. The Marshmallow Test duration was not significantly associated 
with any of the covariates. Maternal parity, gestational diabetes mellitus (GDM), concurrent depressive symptoms, and child's gestational age, standard deviation scale (SDS) birth weight, child's SDS height, and SDS BMI at follow-up were not associated with any child neurodevelopmental measure. Maternal prenatal SO was not associated with maternal smoking, parity, concurrent anxiety symptoms, foreign language status, child's sex, gestational age, or SDS height at follow-up. As maternal parity, gestational age, and children's SDS height were not associated with maternal obesity or any neurodevelopmental outcomes, they were excluded from the regression models. Among the continuous child neurodevelopmental measures, higher HTKS scores were correlated with higher ASQ sum-scores $(r=0.37, P=0.002)$, whereas the Marshmallow Test duration was not associated with the other neurodevelopmental test or questionnaire scores.

\section{The Associations Between Maternal Prenatal SO and Child Neurodevelopment}

Supplementary Table S3 describes the outcomes of neurodevelopmental tests according to maternal SO status. Table 2 presents the results of the linear regressions on maternal prenatal SO and child neurodevelopment, and Table 3 presents the results of the logistic regressions on the individual ASQ scales. In model 1 (Table 2), children born to mothers with prenatal SO had significantly lower ASQ sum-scores, lower HTKS scores, and marginally poorer self-control (shorter duration of gratification delay) in the Marshmallow Test than children born to lean mothers, indicating slower neurodevelopment and poorer executive functioning. On the ASQ subscales (model 1, Table 3), maternal prenatal SO was associated with an increased risk of child neurodevelopmental difficulties in fine motor and problem-solving development.

In model 2 (Tables 2 and 3), the effects of maternal prenatal SO on lower HTKS scores and a higher risk of difficulties in ASQ fine motor and problem-solving scales remained significant, whereas those on the ASQ sum-score were marginally significant. In model 3, adjusted for maternal concurrent psychological well-being, maternal prenatal SO was also a significant predictor of lower ASQ sum-score, lower HTKS score, and an increased risk of neurodevelopmental difficulties in ASQ fine motor and problem-solving scales. In model 4 (Tables 2 and 3), the association between maternal SO and lower ASQ sum-score was marginally significant, but maternal SO remained a significant predictor of lower HTKS scores and an increased risk of ASQ problem-solving and fine motor difficulties. The marginal association of maternal SO with poorer self-control in the Marshmallow Test present in model 1 was no longer evident in models 2-4. Finally, in model 5 (Tables 2 and 3), we found no significant associations between maternal prenatal SO and child neurodevelopment, although maternal SO became marginally associated with poorer self-control in the Marshmallow Test.

Notably, in model 5, lower maternal education level was only significantly associated with a higher risk of ASQ

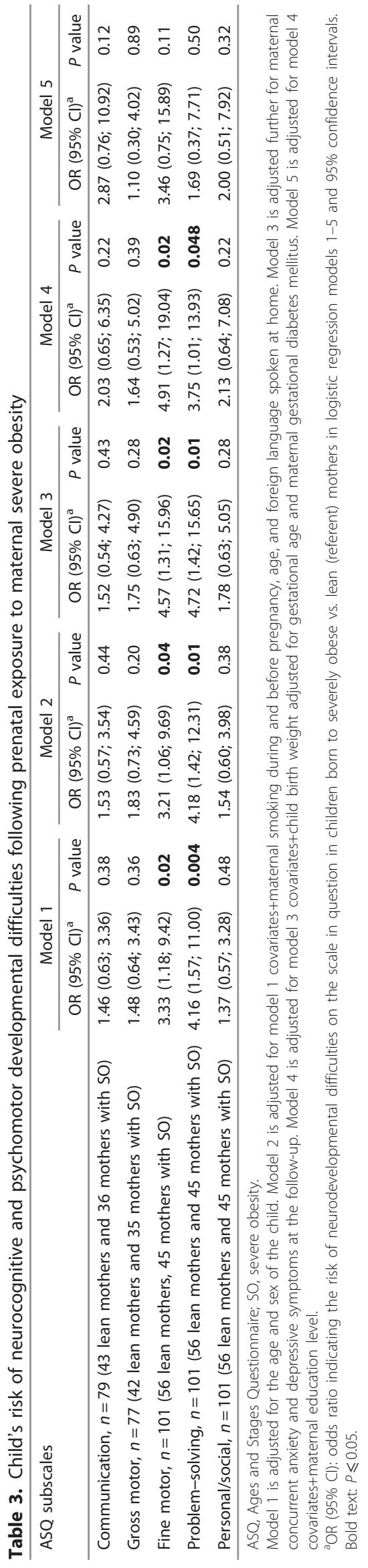




\section{Articles | Mina et al.}

problem-solving difficulties (odd ratio $=1.50,95 \%$ confidence interval $=0.05-0.96, P=0.044$ ), but not with other outcomes $(P \geqslant 0.11)$. As maternal SO status and education level were highly multicollinear $(\varphi=-0.52, P<0.001)$, the changes in the effect of maternal prenatal SO on child neurodevelopmental outcomes in model 5 may be due to the high multicollinearity between maternal obesity and education level, possibly biasing the regression coefficients in these models. Subgroup analyses among children whose mothers attended university education revealed that, although not statistically significant, children prenatally exposed to maternal SO had lower child ASQ total sum-scores, lower subscale scores in gross motor and problem-solving, lower HTKS scores, and poorer self-control in the Marshmallow Test (Supplementary Table S4). After adjusting for multiple confounders, maternal SO did not significantly predict lower subscale scores in the subgroup (data not shown). Taking into account the possible floor and ceiling effects on each scale, Tobit regressions (Supplementary Table S5) yielded mostly corresponding findings with the linear regressions (Table 2). In the Tobit regression models, maternal prenatal SO predicted significantly lower child ASQ total sum-scores in models 1, 3, and 4, lower HTKS scores in models 1-4, and marginally lower ASQ scores in model 2. Similarly, children born to mothers with prenatal SO also had marginally poorer self-control in models 1 and 5, but not in models 2-4 (Supplementary Table S5).

\section{DISCUSSION}

Combining both experimental and questionnaire-based measures of childhood neurocognitive development, the findings of this study support prenatal exposure to maternal obesity as a risk factor for neurodevelopmental delay and worse neurocognitive outcomes. Prenatal exposure to maternal SO predicted poorer neurodevelopment particularly in problem-solving ability and fine motor skills, and also predicted poorer executive functioning skills of attention, inhibitory control, and working memory. The maternal SO effect on the delay of self-gratification, a previously unstudied executive functioning, was marginal and rendered nonsignificant after adjustment for other perinatal factors.

The higher risks of difficulties in ASQ problem-solving skills in children born to mothers with SO are consistent with lower intelligence quotient scores (7-10), a higher risk of intellectual disability $(5,6)$, and lower cognitive performance (16-18) following exposure to maternal obesity. The lower sum-scores of ASQ among children born to obese mothers, implying slower neurodevelopment, support results from studies showing a higher risk of neurodevelopmental delay in children born to obese mothers $(12,15)$. Unlike Hinkle et al. (18), in the current study there was a higher risk of developmental difficulties in fine motor skills among children exposed to prenatal SO. Although poorer gross motor development (34) and higher language impairment (35) among children exposed to maternal obesity have been reported, prenatal exposure to maternal SO did not appear to affect gross motor or communication skills in this study.

In previous studies, maternal overweight did not significantly affect children's neurocognitive outcomes (19), but maternal SO was associated with poorer neurodevelopment of the child (33) as in this study. Further studies are needed to determine whether there is a dose-response or dose-saturation effect of maternal obesity on neurodevelopment.

The results of the HTKS test are in line with the findings from a study using the Go/no-go task (20) and another using the Wisconsin Card Sorting Test and Trail-Making Test (10), each suggesting impaired executive functioning in the areas of attention, self-regulation, inhibition, and working memory following prenatal exposure to maternal obesity. Although the associations between maternal prenatal $\mathrm{SO}$ and poorer selfcontrol in the Marshmallow Test were marginal, the direction of the effect size was consistent with the results from ASQ and HTKS and with our hypothesis that children born to mothers with SO are less able to delay self-gratification for a snack reward. In previous studies, obese (36), but not overweight (37), children did worse in the delay of self-gratification test as compared with children having normal weight. In this study, adjustment for current BMI did not change the results, and, indeed, few children in this study were clinically obese.

As the results of Tobit regressions were similar to those of the linear regressions, the findings in the Marshmallow Test, HTKS Test, and ASQ questionnaires did not appear to be because of the ceiling effects or possible binomial distribution, as suggested by a previous study (38). The marginal findings in the Marshmallow Test could potentially be due to a slight loss of statistical power, as 'non-compliant' participants were excluded from the analysis. It is also possible that the ability to delay gratification, which is one of the most complex executive functioning, is not yet refined in this age group; thus, any difference in performance arising because of prenatal exposure to maternal SO is yet to be observed (27). It is yet to be discovered whether children of mothers with SO will be impaired in their ability to delay self-gratification as they become older.

The overall effects of maternal SO on child neurodevelopment were largely independent of maternal psychological well-being and the child's own obesity. Although familial confounding could not be ruled out as no genetic or sibling/ twin data were available, the findings correspond to previous studies that found no effect of maternal psychological wellbeing on the associations between maternal obesity and children's neurodevelopment outcomes $(20,28)$. This is perhaps unsurprising because the evidence for the associations between maternal prenatal psychological distress and children's cognition has been inconsistent (24-26,39). Although childhood obesity is associated with slower neurocognitive development (27), previous studies have also reported inconsistent findings on the effect of child's BMI $(17,28)$.

The high multicollinearity between maternal education and maternal SO status is challenging, as maternal education is 


\section{Prenatal obesity and neurodevelopment Articles}

also an independent risk factor for increased neurodevelopmental problems in childhood $(5,17,28)$. This multicollinearity is largely contributed by the clear-cut division of maternal education levels according to maternal SO status, where in this sample almost all lean mothers attended higher education (university). Because of this multicollinearity, we were unable to assess whether maternal educational status or maternal SO was a more potent predictor of child neurodevelopment and executive functioning. The subgroup analysis among children whose mothers attended university suggests that the effect of maternal SO may be independent of maternal educational status. However, because of a very small number of children of mothers with SO who attended university, maternal SO did not emerge as a significant predictor of poorer neurodevelopment. Further investigation in a sample with higher proportion of mothers with higher education and obesity is required to determine which factor (obesity vs. education) better predicts child neurodevelopment.

Consistent evidence from animal studies demonstrates that maternal obesity is associated with altered brain development affecting neurocognitive functions in learning and memory through various biological mechanisms involving leptin, insulin, brain-derived neurotrophic factor, and cytokines (40). Very SO increases the risk for GDM and large birth weight, which could also be the causal pathway, but as our main hypothesis focused on maternal $\mathrm{SO}$ and not on the causal factors arising because of maternal obesity, GDM and child's birth weight were included in the analyses as the confounders only. Few human studies have explored biological mechanisms for altered brain development, although deficiencies in micronutrients could possibly affect brain development and consequently offspring neurodevelopment (41). Mothers with SO reported lower micronutrient intake during pregnancy compared with lean mothers (42). Mothers with SO also had altered fatty-acid intake ratio (saturated vs. non-saturated) and lower rates of breastfeeding, both of which have been associated with lower ASQ sumscore (43). Brain development could also be altered by overexposure to maternal inflammation $(44,45)$. More studies are required to understand the biological mechanisms underlying the associations found.

The strengths of this study include the objectively measured maternal BMI and the concurrent administration of developmental screening questionnaires and individually administered tests of executive functioning. We were able to examine the contributions of multiple possible covariates on the associations between maternal SO and child neurodevelopment including prenatal exposure to maternal smoking and GDM, the use of foreign language, maternal concurrent psychological well-being, and children's birth weight and BMI. The limitations include the relatively small sample size to distinguish the effect of maternal lower education from that of maternal prenatal SO in determining children's neurodevelopmental outcomes, the case-control study that might have limited the extrapolation for other groups, and limited correlates of socioeconomic status as maternal income was not directly assessed.

\section{CONCLUSION}

Prenatal exposure to maternal SO is a potential risk factor for delayed neurocognitive development, particularly in areas of problem-solving and fine motor skills and worse executive functioning in the areas of self-regulation, attention working memory, and inhibitory control. It is necessary to determine whether perinatal intervention and management of maternal obesity could alleviate and prevent adverse outcomes in children's neurodevelopment.

\section{SUPPLEMENTARY MATERIAL}

Supplementary material is linked to the online version of the paper at http://www.nature.com/pr

\section{ACKNOWLEDGMENTS}

We thank the mother-and-baby participants and the pediatric nurse team especially Joan Thomson in the Clinical Research Facility, The Royal Hospital for Sick Children, NHS Lothian.

\section{STATEMENT OF FINANCIAL SUPPORT}

We thank Tommy's the Baby Charity for the generous funding. THM received a Principal Development Scholarship, a Charles Darwin Scholarship, and a Global Research Scholarship, University of Edinburgh, Scotland. $M L$ is supported by the Academy of Finland and University of Helsinki Funds. AJD received a Scottish Senior Clinical Fellowship (SCD/09). THM, AJD, and RMR acknowledge the support of the British Heart Foundation.

Disclosure: The authors declare no conflict of interest.

\section{REFERENCES}

1. Heslehurst N, Rankin J, Wilkinson JR, Summerbell CD. A nationally representative study of maternal obesity in England, UK: trends in incidence and demographic inequalities in 619323 Births, 1989-2007. Int J Obes 2010;34:420-8.

2. Weng SF, Redsell Sa, Swift Ja, Yang M, Glazebrook CP. Systematic review and meta-analyses of risk factors for childhood overweight identifiable during infancy. Arch Dis Child 2012;97:1019-26.

3. Casey B, Somerville L, Gotlib I, et al. Behavioral and neural correlates of delay of gratification 40 years later. Proc Natl Acad Sci USA 2011;108: 14998-5003.

4. Kilgour A, Starr J, Whalley L. Associations between childhood intelligence (IQ), adult morbidity and mortality. Maturitas 2010;65:98-105.

5. Heikura U, Taanila A, Hartikainen A, et al. Variations in prenatal sociodemographic factors associated with intellectual disability: a study of the 20-year interval between two birth cohorts in Northern Finland. Am J Epidemiol 2008;167:169-77.

6. Mann J, McDermott S, Hardin J, Pan C, Zhang Z. Pre-pregnancy body mass index, weight change during pregnancy, and risk of intellectual disability in children. BJOG 2013;120:309-19.

7. Neggers Y, Goldenberg R, Ramey S, Cliver S. Maternal prepregnancy body mass index and psychomotor development in children. Acta Obstet Gynecol Scand 2003;82:235-40.

8. Gage S, Lawlor D, Tilling K, Fraser A. Associations of maternal weight gain in pregnancy with offspring cognition in childhood and adolescence: findings from the avon longitudinal study of parents and children. Am J Epidemiol 2013;177:402-10.

9. Huang L, Yu X, Keim S, Li L, Zhang L, Zhang J. Maternal prepregnancy obesity and child neurodevelopment in the Collaborative Perinatal Project. Int J Epidemiol 2014;43:783-92. 
10. Pugh S, Richardson G, Hutcheon J, et al. Maternal obesity and excessive gestational weight gain are associated with components of child cognition. J Nutr 2015;145:2562-9.

11. Hinkle S, Schieve L, Stein A, Swan D, Ramakrishnan U, Sharma A. Associations between maternal prepregnancy body mass index and child neurodevelopment at 2 years of age. Int J Obes 2012;36:1312-9.

12. Krakowiak P, Walker CK, Bremer AA, et al. Maternal metabolic conditions and risk for autism and other neurodevelopmental disorders. Pediatrics 2012;129:e1121-8.

13. Craig W, Palomaki G, Neveux L, Haddow J. Maternal body mass index during pregnancy and offspring neurocognitive development. Obstet Med 2013;6:20-5.

14. Casas M, Chatzi L, Carsin A, et al. Maternal pre-pregnancy overweight and obesity, and child neuropsychological development: two southern european birth cohort studies. Int J Epidemiol 2013;42: 506-17.

15. O'Connor Duffany K, McVeigh K, Kershaw T, Lipkind H, Ickovics J. Maternal obesity: risks for developmental delays in early childhood. Matern Child Health J 2016;20:219-30.

16. Basatemur E, Gardiner J, Williams C, Melhuish E, Barnes J, Sutcliffe A. Maternal prepregnancy BMI and child cognition: A longitudinal cohort study. Pediatrics 2013;131:56-63.

17. Tanda R, Salsberry P, Reagan P, Fang M. The impact of prepregnancy obesity on children's cognitive test scores. Matern Child Health J 2013;17: 222-9.

18. Hinkle S, Sharma A, Kim S, Schieve L. Maternal prepregnancy weight status and associations with children's development and disabilities at kindergarten. Int J Obes 2013;37:1344-51.

19. Brion $M$, Zeegers $M$, Jaddoe $V$, et al. Intrauterine effects of maternal prepregnancy overweight on child cognition and behavior in 2 cohorts. Pediatrics 2011;127:e202-11.

20. Buss C, Entringer S, Davis E, et al. Impaired executive function mediates the association between maternal pre-pregnancy body mass index and child ADHD symptoms. PLoS ONE 2012;7:e37758.

21. Seeyave D, Coleman S, Appugliese D, et al. Ability to delay gratification at age 4 years and risk of overweight at age 11 years. Arch Pediatr Adolesc Med 2009;163:303-8.

22. Molyneaux E, Poston L, Ashurst-Williams S, Howard LM. Obesity and mental disorders during pregnancy and postpartum. Obstet Gynecol 2014;123:857-67.

23. Mina T, Denison F, Forbes S, Stirrat L, Norman J, Reynolds R. Associations of mood symptoms with ante- and postnatal weight change in obese pregnancy are not mediated by cortisol. Psychol Med 2015;45: 3133-46.

24. Buss C, Davis E, Hobel C, Sandman C. Maternal pregnancy-specific anxiety is associated with child executive function at 6-9 years age. Stress 2011;14:665-76.

25. Pearson R, Bornstein M, Cordero M, et al. Maternal perinatal mental health and offspring academic achievement at age 16: the mediating role of childhood executive function. J Child Psychol Psychiatry 2016;57: 491-501.

26. Ibanez G, Bernard J, Rondet C, et al. Effects of antenatal maternal depression and anxiety on children's early cognitive development: A prospective cohort study. PLoS ONE 2015;10:e0135849.
27. Liang J, Matheson B, Kaye W, Boutelle K. Neurocognitive correlates of obesity and obesity-related behaviors in children and adolescents. Int J Obes 2014;38:494-506.

28. Eriksen H, Kesmodel U, Underbjerg M, Kilburn T, Bertrand J, Mortensen E. Predictors of intelligence at the age of 5: Family, pregnancy and birth characteristics, postnatal influences, and postnatal growth. PLoS One 2013;8:1-8.

29. Mischel W, Shoda Y, Rodriguez MI. Delay of Gratification in Children. Science 1989;244:933-8.

30. Kidd C, Palmeri H, Aslin R. Rational snacking: Young children's decision-making on the marshmallow task is moderated by beliefs about environmental reliability. Cognition 2013;126:109-4.

31. McClelland M, Cameron C, Duncan R, et al. Predictors of early growth in academic achievement: The head-toes-knees-shoulders task. Front Psychol 2014;5:1-14.

32. Hornman J, Kertjens JM, de Winter AF, Arend BF, Reijneveld SA. Validity and internal consistency of the Ages and Stages Questionnaire 60 -month version and the effect of three scoring methods. 2013Early Human Dev 89:1011-5.

33. Jo H, Schieve L, Sharma A, Hinkle S, Li R, Lind J. Maternal prepregnancy body mass index and child psychosocial development at 6 years of age. Pediatrics 2015;135:e1198-209.

34. Wylie A, Sundaram R, Kus C, Ghassabian A, Yeung E. Maternal prepregnancy obesity and achievement of infant motor developmental milestones in the upstate KIDS study. Obesity 2015;23:907-13.

35. Whitehouse A, Shelton W, Ing C, Newham J. Prenatal, perinatal, and neonatal risk factors for specific language impairment: a prospective pregnancy cohort study. J Speech Lang Hear Res 2014;57:1418-27.

36. Bonato P, Boland FJ. Delay of gratification in obese children. Addict Behav 1983;8:71-4.

37. Bourget V, White DR. Performance of overweight and normal-weight girls on Delay of gratification Tasks. Int J Eat Disord 1984;3:63-71.

38. Francis L, Susman E. Self-regulation and rapid weight gain in children from age 3 to 12 years. Arch Pediatr Adolesc Med 2009;163:297-302.

39. Keim S, Daniels J, Dole N, Herring A, Siega-Riz A, Scheidt P. A prospective study of maternal anxiety, perceived stress, and depressive symptoms in relation to infant cognitive development. Early Hum Dev 2011;87:373-80.

40. Cordner Z, Tamashiro K. Effects of high-fat diet exposure on learning \& memory. Physiol Behav 2015;152:363-71.

41. Lam L, Lawlis T. Feeding the brain - The effects of micronutrient interventions on cognitive performance among school-aged children: a systematic review of randomized controlled trials. Clin Nutr 2016. (doi:10.1016/j.clnu.2016.06.013).

42. Mohd-Shukri N, Duncan A, Denison F, et al. Health behaviours during pregnancy in women with very severe obesity. Nutrients 2015;7:8431-43.

43. Bernard J, de Agostini M, Forhan A, et al. The dietary n6:n3 fatty acid ratio during pregnancy is inversely associated with child neurodevelopment in the EDEN mother-child cohort. J. Nutrition 2013;143:1481-8.

44. Bilbo S, Schwarz J. The immune system and developmental programming of brain and behavior. Front. Neuroendocrinol 2012;33:267-86.

45. Brown AS, Sourander A, Hinkka-Yli-Soumaki S, McKeague IW, Sundvall J, Surcel HM. Elevated maternal C-reactive protein and autism in a national birth cohort. Mol Psychiatry 2014;19:259-64. 